\title{
Tests of an omnipresent standard for absolute spectral radiance measurements
}

\author{
A. Migdalla, ${ }^{a}$, E. Dauler ${ }^{\mathrm{a}}$, A. Muller ${ }^{\mathrm{b}}$, A. Sergienko ${ }^{\mathrm{b}}$ \\ ${ }^{a}$ Optical Technology Division, 221/B208 National Institute of Standards and Technology, Gaithersburg, MD 20899, USA \\ ${ }^{\mathrm{b}}$ Department of Electrical and Computer Engineering, Boston University, 44 Cummington St., Boston, MA 02215, USA
}

Received 30 June 1998; accepted 26 September 1998

\begin{abstract}
Correlated pairs of photons can be used to access what is effectively an omnipresent absolute standard of spectral radiance. The process of spontaneous parametric downconversion, in which pump photons are converted into pairs of photons, can be thought of as being stimulated by an omnipresent and omnidirectional one photon per mode vacuum background. This background has units of spectral radiance and can be expressed in terms of fundamental constants. An unknown radiance can be determined by comparison to this background radiance. The comparison is made by adding the unknown radiance into downconversion process so as to stimulate the downconversion process over that level produced by the vacuum background only. This is done by inputting the unknown radiance into the system so as to overlap spatially and spectrally a portion of the output light. The process is monitored, not by observing the input light beam, but by observing only the light correlated to that unknown radiance. (This makes possible an additional advantage of this measurement technique; it allows IR radiance to be measured by monitoring a visible beam.) The ratio of the increase in the correlated signal is the absolute spectral radiance of the unknown source expressed in units of photons per mode. Initial studies of feasibility and accuracy have been performed. IR radiance has been measured to wavelengths of $5 \mu \mathrm{m}$ with better than $3 \%$ uncertainty. We present the status of work to further improve the uncertainty of this method. (C) 1999 Elsevier Science B.V. All rights reserved.
\end{abstract}

Keywords: Spectral radiance; Standard

\section{Introduction}

We are investigating a technique that allows spectral radiance to be measured by direct comparison to what is effectively an omnipresent primary standard. By this, we mean a source of spectral radiance that can be thought of as due to a background of zero-point fluctuations in the vacuum field and is thus available

\footnotetext{
*Corresponding author. Tel.: +301-975-2331; fax: +301-9752950; e-mail: amigdall@nist.gov
}

everywhere and to everyone. It is a primary standard because the spectral radiance of this background can be calculated from fundamental constants and thus needs no external calibration.

A major advantage of an omnipresent standard is that it allows a very short calibration chain between primary standard and the final device to be measured. This is important because the accuracy of many types of measurements suffer more from the length of the measurement chain than the uncertainty of the primary standard to which the measurement chain is anchored. 
Accessible primary standards are one way to address this problem, allowing for higher accuracy measurements even though the primary standard used may not have the least possible uncertainty. The ultimate in this approach is to make measurements by direct comparison to an absolute standard with no intermediary transfer standards. We have applied this approach to the field of spectral radiance. We discuss a technique that allows spectral radiance to be measured by direct comparison to the ultimate inaccessible primary standards, an omnipresent primary standard.

This technique, first proposed by Klyshko in 1977 [1] and demonstrated early on by Kitaeva et al. [2] and Malygin et al. [3], has only recently been verified metrologically [4]. Here we explore how varying several operating parameters affect the uncertainty of the method, yielding guidelines for improving the overall measurement uncertainty. These explorations are crucial for the development of a practical technique that can be used with confidence in a variety of measurement situations.

\subsection{Basic method}

We present only a brief description of the principles here, as more detailed descriptions have already been reported [4,5]. The method makes use of optical parametric amplification, that has the peculiar characteristic that an output is produced even with no apparent input [6]. This output can be thought of as being an amplification of a one-photon-per-mode vaccum background that is always present. Because, this background quantity, "one-photon-per-mode", has the units of spectral radiance and can be written in terms of fundamental constants as $R_{\mathrm{vac}}=h c^{2} / \lambda^{5}$ $[1,7]$, it can be thought of as a primary spectral radiance standard. Being a property of the vacuum, it is also omnipresent. Spectral radiance is measured by comparing the output levels of an optical parametric amplifier when amplifying the radiance to be measured versus amplifying the one-photonper-mode radiance of the omnipresent primary standard.

Our implementation of the measurement method is shown in Fig. 1(a). The optical parametric amplifier consists of a nonlinear crystal pumped by a laser at wavelength $\lambda_{\mathrm{p}}$. A noncollinear optical parametric downconversion (PDC) arrangement is used, produ-

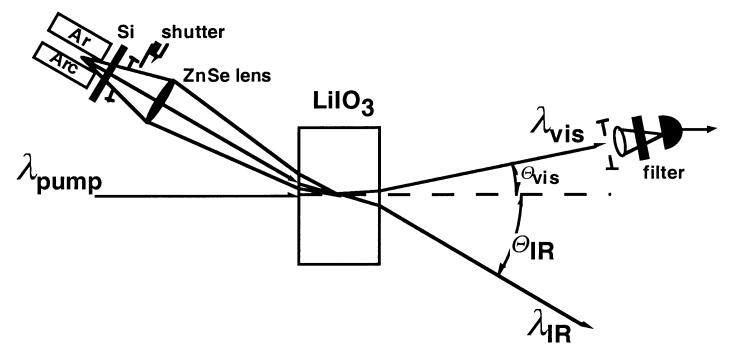

(a.)

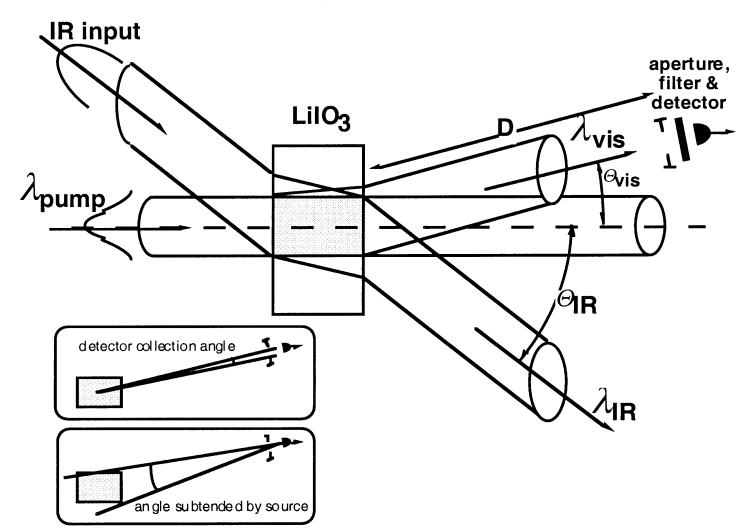

(b.)

Fig. 1. (a) Scheme for absolute radiance measurement using parametric downconversion. (b) Details of the intersection of pump and IR input beams and the detection viewing direction.

cing pairs of downconverted photons, emitted over a range of wavelengths and output angles determined by the constraints of energy conservation and phase matching [8]. In our setup one photon will be in the visible, $\lambda_{\text {vis }}$, and the other of the pair will be in the IR,

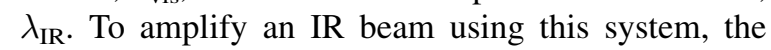
beam is inserted into the crystal so as to overlap (spatially, spectrally and angularly) a portion of the downconverted output. Because the output photons are created in pairs, this amplifier actually has two output channels, one at the wavelength and direction of the input beam and one at the wavelength and direction of the photons correlated to that input beam (as determined by the energy and phase matching constraints). This allows one to use a visible detector to monitor an IR beam. The spectral radiance of an IR beam may be obtained from the ratio of the visible output of the amplifier when amplifying the beam to be measured versus amplifying just the background radiance. Because the absolute spectral radiance deter- 
mination depends only on the ratio of the visible signal levels rather than any absolute light level, a calibrated detector is not required; it need only be linear.

The key to making this technique metrologically robust lies in understanding the sensitivity of the amplification process to spatial, angular, and spectral effects. These, in turn, determine the spatial, angular, and spectral ranges that must be filled by the radiance beam to be measured. Ideally these ranges would be uniformly overfilled. In practice this cannot be achieved. However, by modeling how well these ranges are filled, one can determine an overlap factor that is used to scale the observed ratio to the ideal ratio (that would be obtained with a uniformly overfilled sensitive region). Because making this overlap factor as close as possible to unity reduces the uncertainty of its calculation, it is important to understand how it depends on various parameters.

The spectral radiance measurement equation as described in the previous work is

$R=\frac{\left(n_{\mathrm{vis}}(\text { on }) / n_{\mathrm{vis}}(\text { off })\right)-1}{\epsilon}$,

where $n_{\mathrm{vis}}($ on $)$ and $n_{\mathrm{vis}}(\mathrm{off})$ are the visible PDC signals with the IR source shutter open and closed, and $\epsilon$ is the total system efficiency [4]. The $\epsilon$ term is the product of the overlap factor and $\tau_{\mathrm{IR}}$, the transmittance of the IR imaging system. ( $\tau_{\mathrm{IR}}$ is used to extract the radiance at the source rather than the radiance as imaged into the crystal.) It is the nature of the overlap component of $\epsilon$ that is the focus of this work.

Here we explore the effects of varying the magnification and collection angle on this overlap factor. By this, we are able to find the optimum configuration that yields the maximum overlap factor thus reducing its uncertainty. In addition, verifying the shape of the overlap dependence on these parameters has provided an additional test of our understanding of the overall method.

\subsection{Overlap model}

\subsubsection{Spatial overlap}

Here we present an overview of a number of effects that are included in our model of the overlap factor. In our original paper we calculated only a spatial overlap with assumptions that that was the most significant component. While that was a reasonable assumption in that particular measurement arrangement, it is useful to quantify and test the limits of that assumption.

The spatial overlap factor is calculated by integrating the product of pump beam and IR beam spatial profiles within the crystal and normalizing to the integral of the pump beam in the same volume. Fig. 1(b) shows schematically the spatial profiles of the beams and the intersection region. The pump beam profile is Gaussian. The IR beam profile was best fit to a parabolic form with a cutoff at zero. An additional refinement has been added to this spatial overlap calculation since the previous work. While the pump beam enters the crystal fairly close to normal, the IR beam enters at a large angle resulting in an astigmatic IR beam within the crystal. This astigmatism is now included in the overlap by using separate factors to describe the horizontal and vertical extents of the beam.

\subsubsection{Angular overlap}

In our original work [4] the angular overlap factor was assumed to be unity. Here we explicitly calculate its value. In analogy to the spatial factor which is the integral of the product of two profiles, here we integrate the product of the angular profile of the IR input beam and the profile of the sensitive IR angular range. The difference for this case is that we determine the sensitive IR angular range by converting from the range of visible angles seen by the detector through the phase matching and energy constraints. This overlap factor also requires a two-dimensional integral, because, while the vertical range of angles seen by the detector is limited solely by the geometric collection limit of the detector (determined by the detector aperture and its distance from the source), the horizontal range may be limited either by the geometry of the detector's collection optics or its spectral limitation. (The spectral filter can limit the range of angles seen though the phasematching constraint that ties the wavelength of the output to its angle of output, see [4] for details.) For the profiles of the vertical and horizontal sensitive IR angular ranges, we have used Gaussian shapes with halfwidths matched to these limits. The angular profile of the arc source output beam was measured experimentally to be relatively uniform over a full angle of ca. $80 \mathrm{mrad}$ and to have a 
full width half maximum of ca. $150 \mathrm{mrad}$ (equivalent to $\mathrm{f} / 6.7)$.

\subsubsection{Detector viewing limit}

A third factor affecting the overlap calculation was also considered. This factor limits the region within the crystal pumped by the laser that may be seen by the detector. This limitation occurs because the detector collection aperture is finite and the crystal is an extended source (see insets of Fig. 1(b)), so there can be arrangements where portions of the crystal emit light of the proper wavelengths to be passed by the spectral filter, but at angles that cannot reach the detector geometrically. This effect is included in the overlap integral by the addition of a third factor. The total spatial overlap then becomes the integral of the product of the pump beam, the IR beam, and the visible viewing region. Because this viewing region limitation samples a relatively broad range of the overlap of the other two profiles, the addition of this third limit has relatively little effect on the final overlap, only slightly raising the overlap factors for the lowest magnifications. The overlap factor increases because the detector preferentially samples the center of the crystal where the overlap of the pump and IR beams is greatest.

Fig. 2 shows how the individual spatial and angular overlaps vary with magnification for a fixed lens aperture. They vary as expected: the spatial overlap factor approaches unity as the magnification increases and the angular overlap approaches one at small magnifications where the angular extent of the beam is greatest. The effect of these two factors is seen in the product, that shows a maximum at an intermediate magnification.

\section{Experimental}

The measurement system consists of a $\mathrm{LiIO}_{3}$ crystal $3 \mathrm{~mm}$ thick pumped by an $\mathrm{Ar}^{+}$laser operating at $457.9 \mathrm{~nm}$, with a beam waist of $0.8 \mathrm{~mm}$ at the crystal. The angle between the crystal optic axis and the pump beam was $26.1^{\circ}$, allowing us to produce $0.506 /$ $4.81 \mu \mathrm{m}$ pairs of downconverted photons at output angles of $3.2^{\circ}$ and $31.7^{\circ}$, respectively. The $0.506 \mu \mathrm{m}$ light was detected by a silicon avalanche photodiode (APD) $560 \mathrm{~mm}$ from the crystal. A $2.9 \mathrm{~nm}$ bandpass filter centered at $0.506 \mu \mathrm{m}$ was used to limit the spectrum of the light seen by the detector. In addition, a $0.4 \mathrm{~mm}$ detector collection aperture was used. As discussed in our previous paper, the range (whether limited by geometric collection angles or a spectral filter bandpass) of visible light seen by the detector determines the ranges of IR radiance to which the measurement is sensitive.

The radiance source measured is an Ar discharge arc with a temperature nominally equivalent to a $10000 \mathrm{~K}$ blackbody [9]. The output of this source is imaged into the crystal with a $101 \mathrm{~mm}$ focal length $\mathrm{ZnSe}$ lens. An iris mounted on the lens is used to set the collection f/\#. An antireflection coated silicon filter eliminates visible and UV output of the arc. A shutter is used to turn the radiance beam from the arc on and off. The arc and imaging optics are all mounted on an optical rail. The end of the rail is attached to a gimbal so that the optical axis of the rail stays centered on the crystal as its angle is varied. This allows the input direction of the IR beam to be adjusted for maximum angular overlap nearly independently of the spatial overlap.

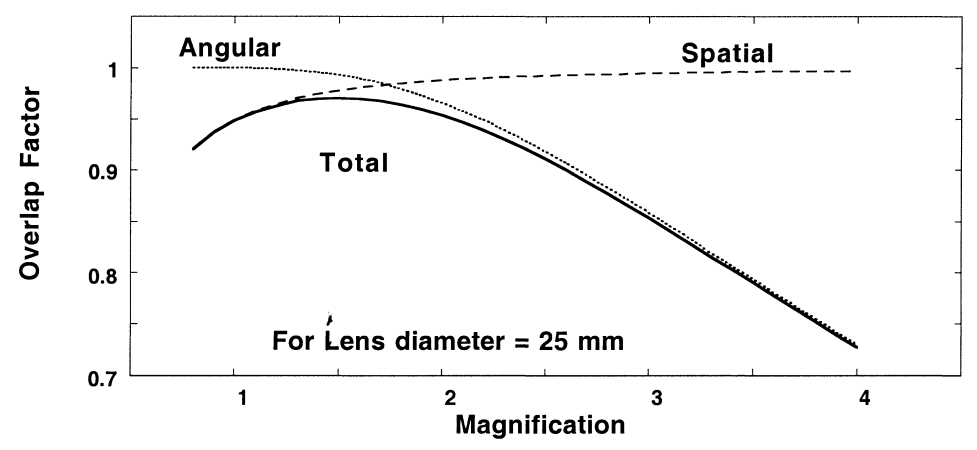

Fig. 2. Calculations of angular, spatial, and total overlap factors are shown for the current experimental setup with a $25 \mathrm{~mm}$ diameter lens aperture. 
Measurements of the ratio of the output at $0.506 \mu \mathrm{m}$ were recorded with the arc shutter open and closed. This ratio was determined as the magnification and lens iris diameters were varied. The magnification was varied from 0.8 to 4 by moving the lens and arc along the optical rail. At each position it was necessary to slightly adjust the horizontal and vertical positions of the lens, to be certain that the IR beam was centered on the crystal region pumped by the laser. For these measurements the transmittance of the IR imaging system $\tau_{\text {IR }}$ was taken to be $0.721 \pm 0.008$ as previously determined.

\section{Results}

Fig. 3 shows the ratios of the downconverted signals of the arc and the background (this ratio must be divided by $\epsilon$ to get the absolute radiance at the source). The ratios are shown for several lens aperture diameters as the imaging magnification is varied. The curves are the result of calculations of the overlap models. The overlap factors are calculated relative to unity, the maximum possible overlap, but for comparison they have been scaled by the radiance of the arc at the crystal as determined previously by conventional means. (The radiance of the arc at the crystal position in the measurement passband is $1.65 \pm 0.03$ photons per mode times $\tau_{\text {IR. }}$.) The data taken with the $25 \mathrm{~mm}$ aperture best show the trade off between the spatial and angular overlap factors as the magnification of the image varies. Large magnification improves the spatial overlap, while lower magnification increases the angular spread of the beam improving the angular overlap factor. The optimum magnification occurs at about 1.5 for the $25 \mathrm{~mm}$ aperture, but moves to smaller magnifications as the aperture is reduced. This occurs because smaller apertures reduce the angular extent of the beam thus reducing the angular overlap factor. By moving to a somewhat smaller magnification with its lower spatial overlap, the angular overlap is increased, optimizing the product of the two factors. Of course this optimum overlap value will be lower than those obtained with a larger lens aperture.

The data are in overall agreement with the overlap modeling. Error bars on the curves indicate uncertainty due to system parameters that are used in calculating the overlap factors. The largest of these is due to the lens diameter. The measured ratios have a dominant relative $5.3 \%$ uncertainty due to source fluctuations (not shown in the figure for clarity). The largest relative deviation between the model and data occurs for the smallest diameter aperture. This is most likely due to uncertainty of the aperture size. For this smallest aperture a fixed diameter uncertainty has the largest relative effect on the overlap calculation. This underscores the point that for lowest uncertainty it is best to operate with the overlap factor as close to one as possible, that is with a large collection angle and optimum magnification.

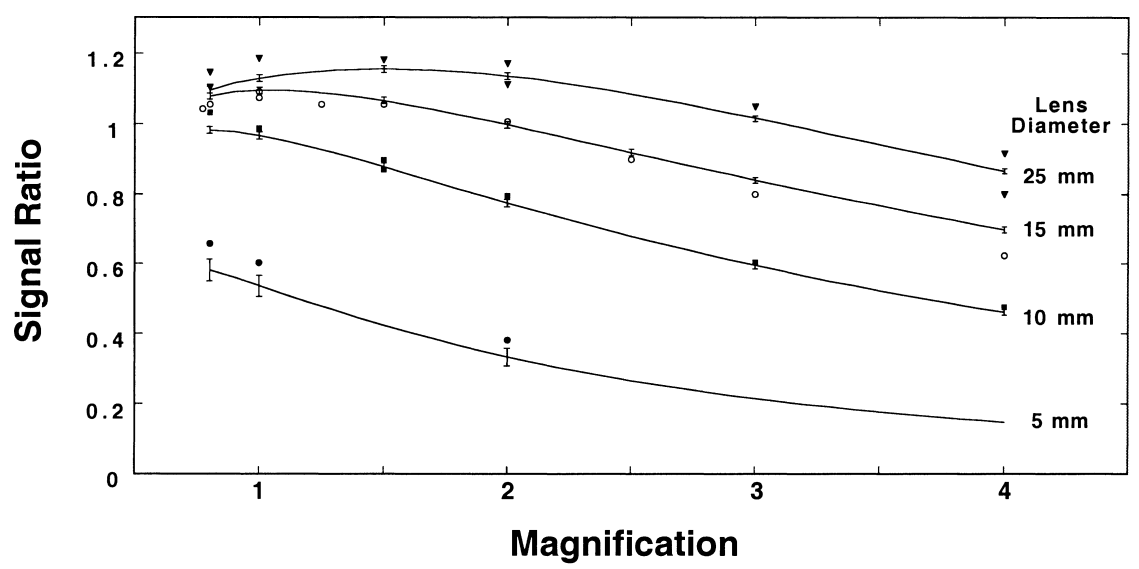

Fig. 3. The points indicate the measured ratios of the signals due to the arc source and the omnipresent background. The curves show results of overlap model calculations. The nominal lens diameters are given for each data set. The circles, boxes, open circles and triangles indicate the $5,10,15$ and $25 \mathrm{~mm}$ data sets, respectively. 


\section{Conclusions}

We have explained how this correlated photon based spectral radiance technique can be considered to be tied to an absolute primary standard that is accessible to all. We have mapped out how the method is affected by varying an important operating parameter, the collection system magnification. We have been able to model these effects indicating good understanding of the underlying processes. This exploration can be used as a guide on how to obtain the optimum signal and lowest uncertainty. These are the keys to making the technique into a truly useful metrological method that can be used with confidence. This should allow others to use this omnipresent spectral radiance standard to take advantage of the resulting shortened measurement chain between primary standard and source to be measured.

With a better understanding of these operating parameters we can begin to explore other parameters such as spectral bandwidth which may lead to further improvements in the system efficiency and its associated uncertainty. A straightforward way of testing this would be to replace the spectral filter with a small spectrometer. In addition to a variable bandwidth this would allow tunability of the wavelength region where the spectral radiance is measured.

\section{References}

[1] D.N. Klyshko, Sov. J. Quant. Elect. 7 (1977) 591.

[2] G.Kh. Kitaeva, A.N. Penin, V.V. Fadeev, Yu.A. Yanait, Sov. Phys. Dokl. 24 (1979) 564.

[3] A.A. Malygin, A.N. Penin, A.V. Sergienko, Sov. Phys. JETP Lett. 33 (1981) 477.

[4] A. Migdall, R. Datla, A. Sergienko, J.S. Orszak, Y.H. Shih, Appl. Opt. (1998), Appl. Opts. 3455 (1998).

[5] E. Dauler, A. Migdall, N. Boeuf, R. Datla, A. Muller, A. Sergienko, Metrologia 35 (1998).

[6] W.H. Louisell, A. Yariv, A.E. Siegman, Phys. Rev. 124 (1961) 1646.

[7] D.N. Klyshko, Photons and Nonlinear Optics, Gordon and Breach, New York, 1988, p. 325.

[8] F. Zernike, J.E. Midwinter, Applied Nonlinear Optics, Wiley, New York, 1973.

[9] J.M. Bridges, A.L. Midgall, Metrologia 32 (1995/96) 625. 